\title{
Um procedimento para alocação de locomotivas a
}

\section{trens de carga}

\section{Sérgio Luiz da Silva Cassemiro ${ }^{1}$, Renata Albergaria de Mello Bandeira ${ }^{2}$, Ulisses da Silva ${ }^{3}$}

${ }^{1}$ Instituto Militar de Engenharia, Rio de Janeiro - Brasil, sergio.cassemiro@engenharia.ufjf.br

2Instituto Militar de Engenharia, Rio de Janeiro - Brasil, re.albergaria@gmail.com

${ }^{3}$ MRS Logística SA, ulissesb.silva@gmail.com

\section{Recebido:}

11 de dezembro de 2017

Aceito para publicação:

23 de agosto de 2019

Publicado:

30 de abril de 2020

Editor de área:

Renato Lima

\section{Palavras-chaves:}

Transporte ferroviário,

Locomotiva,

Alocação.

Keywords:

Rail transport,

Locomotive,

Allocation.

DOI:10.14295/transportes.v28i1.1548

\section{RESUMO}

Este artigo apresenta um procedimento para alocação de diferentes locomotivas em diversos trens de carga. O procedimento é proposto passo a passo, incluindo o levantamento de informações e dados necessários e, por fim, um modelo de otimização com objetivo de minimizar os custos operacionais das locomotivas alocadas, focando em consumo de combustível (eficiência energética) e custos de manutenção. $O$ trabalho apresenta uma aplicação do procedimento proposto com base nos dados de uma empresa ferroviária brasileira de transporte de cargas, demonstrando a oportunidade nas decisões de alocação das locomotivas disponíveis, gerando ganhos de eficiência energética, redução dos custos de manutenção e outros ganhos indiretos.

\section{ABSTRACT}

This article introduces an allocation procedure for different locomotives in different types of cargo trains. It presents a step by step procedure with all the necessary information and required data. In the final step, it presents an optimization model to minimize the operational costs of the locomotives. The cost reduction model focuses on fuel consumption (energy efficiency) and maintenance costs. The paper also presents a practical application based on a Brazilian cargo railway company data, demonstrating the potential benefits in energy efficiency, maintenance costs reduction and other indirect gains.

\section{INTRODUCÃO}

0 modo ferroviário de carga, caracterizado pelo transporte de elevados volumes por trechos geograficamente distantes, se destaca devido a sua alta eficiência energética, apresentando consumo de combustível relativamente baixo em relação ao transporte rodoviário (Magalhães, 2001). Porém, o transporte ferroviário apresenta custos de ativos e de operação relativamente altos conforme VALEC (2012). Estes custos operacionais são diretamente influenciados pela alocação dos ativos, especialmente das locomotivas, de modo que a gestão eficiente destes recursos se apresenta como uma alternativa para melhoria da eficiência e redução de custos.

A atividade de planejamento das locomotivas envolve a solução para as seguintes questões (Gualda e Murgel, 1999): (i) quais locomotivas devem ser alocadas para tracionar quais trens?; (ii) quais locomotivas devem ser rebocadas?; (iii) quais locomotivas devem viajar sem carga?; e, (iv) dentre as opções de trens, onde alocar os diversos tipos considerando-se capacidade, eficiência, tecnologia embarcada, características físicas e restrições do sistema? Enfim, trata-se de 
um desafio que requer amplo planejamento nos níveis estratégico, tático e operacional, configurando-se como um dos principais fatores para tornar as ferrovias mais eficientes. Contudo, não existe certeza e nem comprovação de que as locomotivas sejam alocadas da maneira mais eficiente pelas empresas ferroviárias no Brasil (Cassemiro, 2017).

Neste contexto, o objetivo deste artigo é resolver o problema de alocação de locomotivas por meio de um procedimento que permita responder à seguinte questão básica: quais locomotivas devem ser alocadas em quais trens? Para tanto, é proposto um modelo matemático com foco na minimização dos custos, baseada em duas grandes variáveis de importância: consumo de diesel (melhoria da eficiência energética) e custos de manutenção. VALEC (2012) afirma que a escolha dessas variáveis se justifica pelo fato de constituírem os principais custos operacionais ferroviários. 0 diferencial do procedimento proposto quando comparado com a literatura existente (Piu e Speranza, 2013; Ahuja et al., 2005; Florian et al., 1976), é a simplicidade da modelagem adotada, o modelo matemático baseado na formulação da Eficiência Energética e a indicação de um roteiro que possibilite a sua aplicação em qualquer ferrovia de carga, não se restringindo ao grau de particularidade e parametrização que as soluções pesquisadas conferem ( $\mathrm{Su}$ et al., 2015, Ahuja et al., 2005; Florian et al., 1976).

A partir desta introdução, o artigo se divide em quatro seções. A Seção 2 apresenta a revisão da literatura sobre o Problema de Alocação de Locomotivas (PAL). A Seção 3 descreve passo a passo o procedimento de alocação proposto e apresenta sua aplicação em uma ferrovia de transporte de cargas. A Seção 4 apresenta a discussão dos resultados, enquanto a Seção 5 traz as conclusões e recomendações finais do trabalho.

\section{REVISÃO DA LITERATURA}

Piu e Speranza (2013) apresentaram uma revisão sistemática da literatura e propuseram uma sugestão de classificação dos vários modelos utilizados para a resolução do PAL. Os trabalhos analisados pelos autores foram desenvolvidos com base em técnicas de Pesquisa Operacional (PO), sendo possível verificar que, com o advento da computação, houve um interesse por parte das ferrovias de carga, principalmente americanas e canadenses, em desenvolver e investir na solução computacional do PAL, buscando otimização de vários aspectos: redução de custos, ciclo operacional, emissões de gases, consumo de combustível e quantidade de ativos usados, aumento do lucro e etc.

Florian et al. (1976) utilizaram a modelagem do problema de rede multicommodity com o objetivo de minimizar o investimento e o custo de manutenção no longo prazo. As restrições foram baseadas nas necessidades de tração e consideraram peso e comprimento do trem, geografia do trajeto, número de vagões, dentre outras. A proposta foi uma solução baseada na Decomposição de Benders para resolver o problema de planejamento num horizonte semanal, sendo aplicado em uma das regiões da empresa ferroviária Canadian National.

Ahuja et al. (2005) apresentaram um estudo na empresa ferroviária CSX, focando em um planejamento semanal da alocação das locomotivas em trens, com objetivo de minimizar custos. A estruturação foi feita sob a ótica de rede de multicommodity, em que cada tipo de locomotiva é tratado como um produto. 0 custo total foi definido com alguns itens diferenciados dos demais trabalhos, como a soma dos custos de propriedade dos ativos, os de viagens sem tracionar e o da capacidade de tração excedente. Foi proposta uma heurística de Neighborhood Search que utiliza estruturas de vizinhança para buscar soluções otimizadas. 
Paoletti e Cappelletti (2007) desenvolveram uma modelagem que aloca as locomotivas segundo a grade de horários dos trens, considerando a visão de programação diária. Cada locomotiva segue um ciclo, realizando a tração de um ou mais trens e retornando ao seu ponto de origem. 0 trabalho envolveu mais de 4.000 locomotivas em 50 tipos diferentes, o que possibilita mais de 200 formações de tração para cerca de 9.000 serviços. 0 problema foi modelado como redes de muticommodity e busca minimizar o custo operacional através de um algoritmo heurístico.

Hu et al. (2013) propuseram um modelo de otimização multiobjetivo (fuzzy) e trabalharam na programação de trens, considerando restrições de emissões de gases e eficiência energética. O objetivo foi reduzir o custo com combustível, emissões e tempo de viagem das locomotivas. A aplicação foi realizada em um trecho pequeno, contendo três estações, três segmentos de linha, três tipos de trens e três tipos de locomotivas com e sem restrições de emissões de gases.

Piu et al. (2015) introduziram primeiramente um conceito de consist como sendo a formação de grupos tratores (blocos de locomotivas) e seu modelo trabalhou na montagem destes blocos de tração antes de alocar nos trens. No segundo estágio, ocorreu a solução do que os autores chamaram de um problema de roteamento, em que o principal objetivo foi alocar estes grupos de tração em uma rede de trens, minimizando os custos. Portanto, introduziram uma forma mais completa, com o modelo de solução dividido em etapas. A base da modelagem é um problema de fluxo em redes multicommodity e, apesar de trabalharem com sete tipos de locomotivas diferentes, os grupos tratores predeterminados (consists) definiam uma commodity.

Su et al. (2015) desenvolveram um modelo de otimização da alocação de múltiplos tipos de locomotivas da ferrovia chinesa Da-quin Railway com objetivo de minimizar a quantidade desses ativos. Os autores conseguiram simplificar o problema de alocação a partir de regras de blocagem predeterminadas pela ferrovia. Com isso, foi aplicado o Algoritmo Húngaro para a obtenção de um resultado exato da alocação na grade padrão de horários.

No Brasil, Louzada et al. (2018) propuseram um modelo matemático de Programação Inteira que se baseia numa estratégia para distribuição de vagões vazios, onde esses são transportados utilizando a folga na capacidade de tração dos trens que circularão com vagões carregados já programados para atendimento às demandas dos pátios. Baseado em dados reais da Ferrovia Centro-Atlântica (FCA), os autores encontraram a solução ótima utilizando o solver CPLEX 12.6.

De forma geral, os modelos abordaram variáveis como tempo de viagem, capacidade de tração, topografia, timetable dos trens, tipos de vagões, entre outras, ao tratar o problema de alocação de locomotivas. Apenas Florian et al. (1976) e Ahuja et al. (2005) trataram o problema com um horizonte de uma semana de planejamento, porém com um alto tempo de processamento. Tampouco foi identificado na revisão da literatura uma diversidade de trabalhos nacionais que utilizam dados reais e solução por otimização. Deste modo, os autores optaram por realizar entrevistas com profissionais envolvidos neste processo em empresas ferroviárias no Brasil e verificaram que, atualmente, o problema é resolvido de forma empírica, sendo que cada ferrovia adota um padrão de alocação sem a utilização de um software de planejamento específico.

Neste contexto, este artigo propõe um modelo matemático que visa maximizar a Eficiência Energética no processo de alocação de locomotivas, além da indicação de um roteiro que possibilite a sua aplicação em qualquer ferrovia de carga, considerando um período de análise mensal ou semanal. 0 roteiro conduz a uma série de simplificações que foram inseridas baseadas 
nas publicações revisadas, destacando Paoletti e Cappelletti (2007) com a introdução do conceito de trens e locomotivas cíclicas, Su et al. (2015) que introduziram regras de blocagem prévias ao modelo e Piu et al. (2015) com o fracionamento do problema em duas partes, blocagem e roteamento. Isso possibilitou simplificar bastante o modelo a ponto de torná-lo mais ágil e fácil de ser utilizado, obtendo uma boa solução em menos de um minuto de processamento computacional. Assim, o procedimento proposto é inovador à medida que traz uma abordagem objetiva e de simples aplicação, de modo a permitir o planejamento para períodos semanais e/ou mensais. Além disso, o modelo proposto se diferencia dos demais, identificados na literatura, devido ao seu foco em maximizar a eficiência energética. Ademais, este procedimento está adaptado à realidade das ferrovias brasileiras, sendo apresentada ainda uma aplicação que garante sua consistência.

\section{PROCEDIMENTO PARA ALOCAÇÃO DE LOCOMOTIVAS: APLICAÇÃO EM UMA FERROVIA BRASILEIRA DE TRANSPORTE DE CARGAS}

O problema de alocação de locomotivas, em sua primeira fase, consiste no planejamento da alocação. Segundo Piu et al. (2015), o objetivo do Problema de Planejamento de Locomotiva (PPL) é definir quais conjuntos de locomotivas (quantidade e frota) são alocadas a cada padrão de trem adotado pela ferrovia. Estes padrões são caracterizados pela sua formação, rota e capacidade, e nas ferrovias são comumente chamados de trem tipo. As etapas seguintes ao PPL, como programação e roteamento, tratam a identificação de cada veículo em particular (não apenas a frota), e as suas atuais condições e características como autonomia, restrições de função, necessidade de manutenção, passagem em oficinas, entre outras.

De forma geral, a integração destas três etapas não é praticável devido ao tamanho e complexidade dos problemas reais. 0 presente trabalho tem como foco a etapa estratégica do planejamento, visto que ela é fundamental para o direcionamento das etapas operacionais, em que decisões em tempo real podem ser tomadas de forma mais facilitada e assertiva quando o PPL é bem resolvido. Portanto, o procedimento proposto se restringe ao PPL em trens com características cíclicas, o que é mais comum em ferrovias de carga. A Figura 1 apresenta as etapas do procedimento aplicado para a solução do planejamento mensal de alocação de locomotivas da MRS Logística, uma ferrovia que atende o sudeste brasileiro e é considerada a maior operadora ferroviária do Brasil. Porém, por questões de sigilo, a empresa ferroviária solicitou que os dados fossem modificados para não expor as informações dos clientes e da ferrovia. A seguir, as etapas do procedimento e os resultados da aplicação são detalhados.

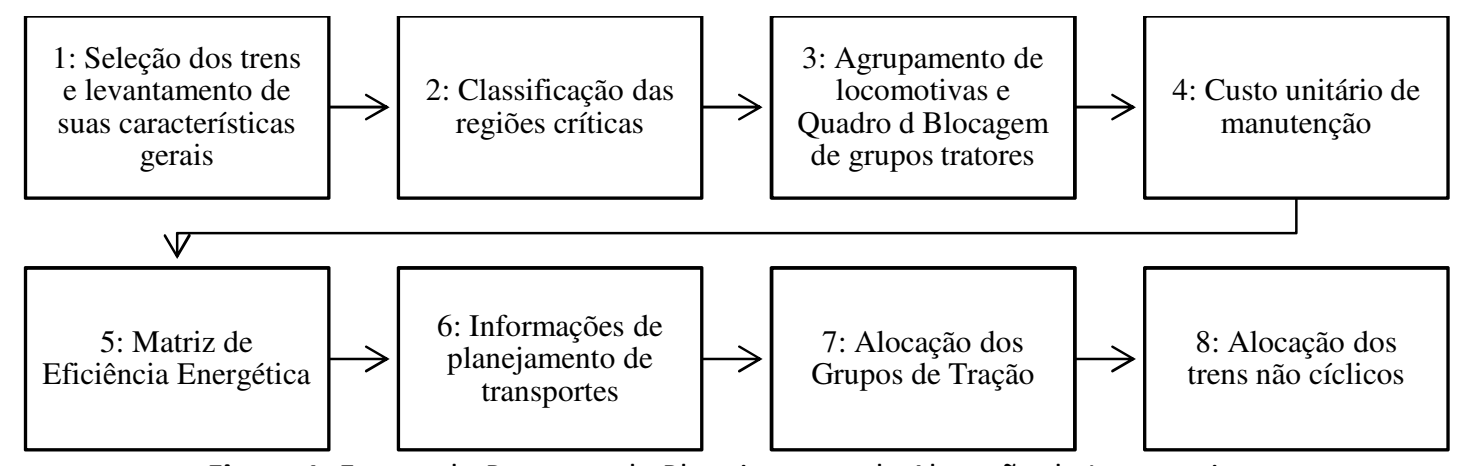

Figura 1. Etapas do Processo de Planejamento de Alocação de Locomotivas 


\subsection{Etapa 1: Seleção dos trens e levantamento de suas características gerais}

Nesta etapa, são levantadas as seguintes informações mensais históricas: origem, destino e rota do trem tipo, sua distância $(\mathrm{km})$ e a tonelada bruta x quilômetro transportada (TKB) por trem tipo. Também é necessário estimar a TKB planejada por trem tipo e a quantidade de composições $\left(\theta_{i}\right)$ a serem utilizadas nos meses que se quer aplicar o procedimento de alocação.

0 conceito de composição, definido por $\theta_{i}$, é quando se agrupa uma quantidade de vagões interligados de uma maneira padrão para determinado trem tipo. Este grupo de vagões faz vários ciclos de viagens, carregando, transportando, descarregando e retornando a origem sucessivas vezes. Em sua grande maioria, são cíclicos, pois eles partem de determinado local e retornam ao mesmo, na maioria das vezes, com a mesma composição. Podem até receber ou trocar locomotivas ou vagões, mas mantêm uma rota padrão, retornando sempre ao mesmo ponto de origem. Sabe-se, por meio da razão entre a capacidade da composição e o tempo para a realização de um ciclo, a quantidade de TKB que um trem é capaz de realizar durante certo período. Neste trabalho, as estimativas foram feitas em períodos mensais.

Para o caso estudado, foi realizado o levantamento de todos os trens elegíveis, totalizando 14 trens tipo. Estes trens representam mais de 90\% do TKB total tracionado em 2016 para a ferrovia analisada. A Tabela 1 apresenta um resumo das características gerais dos trens selecionados. 0 índice $i$ é apenas a identificação de trem tipo que será utilizada mais à frente.

\subsection{Etapa 2: Classificação das regiões críticas}

A Etapa 2 consiste no levantamento dos dados de via permanente para a classificação das regiões críticas nas rotas dos trens. Estes levantamentos são realizados em campo por equipes de topografia, e em geral, as ferrovias possuem os perfis longitudinais das rampas e curvas representados de forma simplificada ou em planilhas eletrônicas. Assim, deve ser feita uma classificação dos perfis de acordo com a faixa de valores de resistências das curvas e das inclinações da via consolidadas representadas como uma inclinação única para facilitar os cálculos de necessidade de tração chamada de rampa compensada. Com isso é possível determinar a região mais crítica do trecho. Para o cálculo da rampa compensada, geralmente, é adotado o comprimento do trem tipo em questão.

Tabela 1 - Resumo dos Trens Selecionados

\begin{tabular}{|c|c|c|c|c|c|c|}
\hline $\bar{i}$ & Trem Tipo & Mercadoria & Origem & Destino & $T K B_{(\text {mensal) }}$ & $\boldsymbol{\theta}_{i}$ \\
\hline 1 & FER & Ferro Gusa & Terminal Sarzedo Novo (MG) & Arará/Porto do Rio de Janeiro (RJ) & 35.007 .783 & 5 \\
\hline 2 & JDN1 & Produtos Agrícolas & Terminal Boa Vista (SP) & Porto de Santos (SP) & 24.420 .296 & 2 \\
\hline 3 & JDN2 & Produtos Agrícolas & Itirapina (SP) & Porto de Santos (SP) & 89.027 .449 & 4 \\
\hline 4 & JDN3 & Produtos Agrícolas & $\begin{array}{l}\text { Porto Hidroviário Pederneiras } \\
\text { (SP) }\end{array}$ & Porto de Santos (SP) & 88.062 .539 & 8 \\
\hline 5 & JDN5 & Produtos Agrícolas & Terminal Pradópolis (SP) & Porto de Santos (SP) & 18.807 .960 & 1 \\
\hline 6 & JDU & Produtos Agrícolas & $\begin{array}{l}\text { Porto Hidroviário Pederneiras } \\
\text { (SP) }\end{array}$ & $\begin{array}{l}\text { Porto de Santos - } \\
\text { Margem Esquerda (SP) }\end{array}$ & 8.018 .884 & 2 \\
\hline 7 & NEXP & Minério de Ferro & Região Paraopeba (MG) & Portos do Rio de Janeiro (RJ) & 6.053 .716 .142 & 62 \\
\hline 8 & NEV & Minério de Ferro & Terminal Casa de Pedra (MG) & Terminal Volta Redonda (RJ) & 151.274 .347 & 4 \\
\hline 9 & AUX-FA & helper & Pátio P1-07 (MG) & Pátio Bom Jardim (MG) & 1.067 .617 .833 & 42 \\
\hline 10 & AUX-SM & helper & Pátio Barra do Piraí (RJ) & Pátio Humberto Antunes (RJ) & 123.413 .500 & 4 \\
\hline 11 & NEZ & Minério & $\begin{array}{l}\text { Terminal Córrego do Feijão } \\
\text { (MG) }\end{array}$ & Terminal Ouro Branco (MG) & 34.031 .091 & 3 \\
\hline 12 & TOD & Bauxita & Pátio Barão de Angra (RJ) & Alumínio (SP) & 57.738 .516 & 4 \\
\hline 13 & HPN & Celulose & Pátio São Silvestre (SP) & Porto de Santos (SP) & 14.532 .954 & 5 \\
\hline 14 & QVL & Carvão Coque & Porto de Sepetiba (RJ) & Terminal Volta Redonda (RJ) & 42.448 .345 & 5 \\
\hline
\end{tabular}


A partir da rampa crítica, realiza-se o dimensionamento da necessidade de tração para o trem tipo. Desta forma, um primeiro critério é realizar a divisão das regiões em segmentos, onde seja possível realizar trocas de locomotivas ou vagões, caso necessárias. Um segundo critério é o da sensibilidade à mudança de perfil. Geralmente, ao se identificar uma rampa muito acentuada, destoando das demais na região, deve-se isolar o trecho, mesmo que pequeno, para mostrar que existe uma alteração pontual, o que pode indicar e favorecer a necessidade do uso de um helper, ou seja, uma locomotiva que entra tracionando uma composição em parte da viagem, quando há dificuldade para a formação padrão do trem vencer as resistências dos trechos. Com isso, é possível saber quais as rampas críticas na rota inteira dos trens e a necessidade de tração a ser usada. A Figura 2 mostra a representação de um quadro com a classificação de rampas por faixa de valores, representada por letras. Além do quadro, apresenta-se o desenho da rota de um trem tipo que circula da direita para a esquerda, onde foram representados os segmentos de rampa compensada e demarcadas as regiões críticas com os retângulos de destaque.

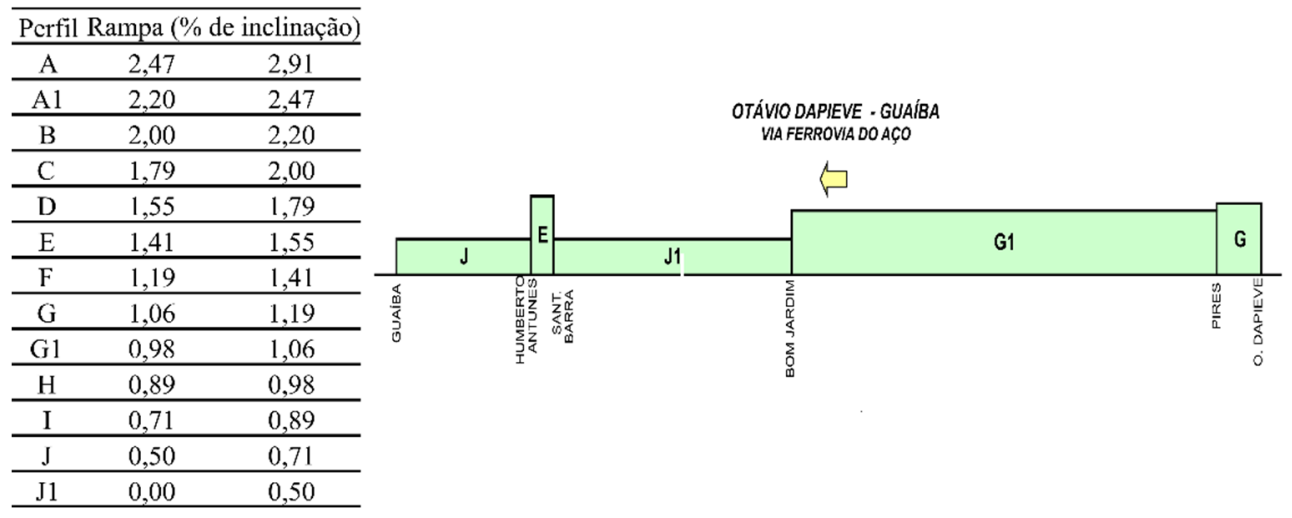

Figura 2. Forma de classificação de rampas e exemplo de rota representada

\subsection{Etapa 3: Agrupamento de locomotivas e Quadro de Blocagem de grupos tratores}

Nesta etapa, afere-se a disponibilidade de cada tipo de locomotiva e as informações dos fabricantes (potência, autonomia, esforço trator e outras). As locomotivas são então agrupadas, considerando suas similaridades. Combinações de locomotivas com características muito diferentes em um mesmo trem geram perdas devido às diferenças de restrições. Por exemplo, uma locomotiva com velocidade mínima de $20 \mathrm{~km} / \mathrm{h}$ restringirá uma outra que poderia chegar a 15 $\mathrm{km} / \mathrm{h}$ oferecendo um esforço trator bem maior do que a de $20 \mathrm{~km} / \mathrm{h}$, gerando um mau aproveitamento de potência e maior consumo de diesel.

Os autores Piu et al. (2015) e Su et al. (2015) obtiveram sucesso em chegar às opções de grupos tratores fracionando o problema com esta etapa (Agrupamento de Locomotivas), sendo a primeira a ser resolvida pelo modelo proposto por eles. Com isso, não sobrecarregaram as decisões ao longo do processo com infinitas opções de cálculos, aumentando a velocidade de processamento e simplificando o modelo. Conforme Piu et al. (2015) e Su et al. (2015), o presente trabalho incorpora essa atividade ao procedimento para ser realizada manualmente e considerando as seguintes informações: esforço trator máximo $\left(E T_{\text {máx }}\right)$, velocidade mínima de regime $\left(\mathrm{Vel}_{\text {mín }}\right)$, potência máxima $\left(P_{\text {máx }}\right)$ e autonomia ou capacidade do tanque de combustível. Destaca-se que, apesar do trabalho prévio de determinação dos blocos ser extenso, ele é feito uma única vez e tem resultado positivo, simplificando o modelo. Com base no quadro de tração 
e no peso de arraste do trem tipo, é criada uma matriz bidimensional onde cada elemento define a quantidade de locomotivas de determinado agrupamento (grupo trator) $j$ necessária para tracionar o trem tipo $i$ em sua rota, baseado no perfil mais crítico. 0 uso de helper pode ser uma boa solução para o problema de tração pontual sem sobrecarregar os trens com locomotivas a mais, que ficarão ociosas pelo resto do percurso. Os helpers, então, são considerados como trens tipo e na Tabela 1 são representados por AUX-FA (auxílio da Ferrovia do Aço) e por AUX-SM (auxílio da Serra do Mar).

A Tabela 2 apresenta o resultado do agrupamento das locomotivas. 0 nome dos grupos foi adotado de forma aleatória, sendo que 10 abrange as mais potentes e que suportam maior esforço, decrescendo a numeração a partir desta lógica. 0 índice $j$ representa a identificação do grupo de locomotiva que será utilizado mais à frente neste trabalho.

Tabela 2 - Resumo do Agrupamento de Locomotivas

\begin{tabular}{|c|c|c|c|c|c|c|}
\hline$j$ & $\begin{array}{l}\text { Grupo } \\
\text { Trator }\end{array}$ & Locomotiva & $\begin{array}{l}P_{\text {máx }} \\
(H P)\end{array}$ & $\begin{array}{c}\text { Capacidade do Tanque } \\
\text { (I) }\end{array}$ & $\begin{array}{l}E T_{\text {máx }} \\
\text { (ton ) }\end{array}$ & $\begin{array}{c}\mathrm{Vel}_{\min } \\
(\mathrm{km} / \mathrm{h})\end{array}$ \\
\hline 1 & 10 & GE-AC44i & 4.400 & 18.000 & 68000 & 15,0 \\
\hline 2 & 9 & GE-C44-EMI & 4.400 & 18.000 & 49546 & 20,0 \\
\hline \multirow{5}{*}{3} & 8 & GE-C36-7 & 3.600 & 15.000 & 40969 & 19,2 \\
\hline & 8 & GE-C36-S7 & 3.600 & 15.000 & 40969 & 19,2 \\
\hline & 8 & GE-MX36-MP & 3.600 & 15.000 & 40969 & 19,2 \\
\hline & 8 & GE-C30-8 & 3.600 & 15.000 & 40969 & 19,2 \\
\hline & 8 & GE-C36-ME & 3.600 & 15.000 & 40969 & 19,2 \\
\hline \multirow{5}{*}{4} & 7 & GE-C30-7 & 3.000 & 15.000 & 40969 & 17,4 \\
\hline & 7 & GE-U30C & 3.000 & 15.000 & 40969 & 17,4 \\
\hline & 7 & GE-C30-7MP & 3.000 & 15.000 & 40969 & 17,4 \\
\hline & 7 & GE-SF30-C & 3.000 & 15.000 & 40969 & 17,4 \\
\hline & 7 & GM-SD40/3 & 3.000 & 15.000 & 40969 & 17,1 \\
\hline
\end{tabular}

O Quadro de Blocagem dos Grupos Tratores, apresentado na Tabela 3, traz o número de locomotivas necessárias para tracionar uma composição de cada trem tipo por sua rota. Essa quantidade foi determinada analisando-se o perfil crítico de cada rota, o peso de cada trem tipo e a capacidade de tração das locomotivas. Por exemplo, o Trem Tipo 1 (FER) pode ser tracionado por duas locomotivas do Grupo Trator 10, ou duas do Grupo 9, ou três do Grupo 8, ou três do Grupo 7.

Tabela 3 - Resumo do Agrupamento de Locomotivas

\begin{tabular}{cccccc}
\hline \multirow{2}{*}{$\boldsymbol{i}$} & \multirow{2}{*}{ Trem Tipo } & \multicolumn{4}{c}{ Grupo Trator } \\
\cline { 3 - 6 } & & $\mathbf{1 0}$ & $\mathbf{9}$ & $\mathbf{8}$ & $\mathbf{7}$ \\
\hline 1 & FER & 2 & 2 & 3 & 3 \\
\hline 2 & JDN1 & 3 & 4 & 5 & 6 \\
\hline 3 & JDN2 & 3 & 4 & 5 & 6 \\
\hline 4 & JDN3 & 3 & 4 & 5 & 6 \\
\hline 5 & JDN4 & 3 & 4 & 5 & 6 \\
\hline 6 & JDU & 2 & 3 & 4 & 5 \\
\hline 7 & NEXP & 2 & 3 & 4 & 4 \\
\hline 8 & NEV & 2 & 3 & 3 & 3 \\
\hline 9 & AUX-FA & 1 & 2 & 2 & 2 \\
\hline 10 & AUX-SM & 2 & 3 & 3 & 4 \\
\hline 11 & NEZ & 2 & 3 & 3 & 4 \\
\hline 12 & TOD & 1 & 2 & 3 & 3 \\
\hline 13 & HPN & 1 & 2 & 2 & 2 \\
\hline 14 & QVL & 2 & 2 & 3 & 3 \\
\hline
\end{tabular}




\subsection{Etapa 4: Custo unitário de manutenção}

As revisões e inspeções de manutenção acontecem periodicamente, mas existem ainda revisões trimestrais, semestrais, anuais, bianuais, trianuais e gerais, cujo indicador de necessidade de manutenção é a quilometragem percorrida ou a quantidade consumida em litros de óleo diesel. Portanto, fatores como extensão do trajeto, tempo de viagem, disponibilidade e utilização da frota afetam diretamente a periodicidade em que a locomotiva vai às oficinas. Este trabalho propõe, por simplificação, a determinação de um custo unitário médio de manutenção $\left(C_{U M}\right)$ por grupo de locomotiva, composto pela soma de todo o custo dado em um ciclo de manutenção pelo fator de consumo de diesel em litros, já que este é o indicador de manutenção, conforme Equação (1). A simplificação se deve ao fato das locomotivas classificadas em um mesmo grupo terem ciclos de manutenção, idade e estado diferentes.

$$
C_{U M}=\frac{\text { Custo de manutenções e revisões durante o ciclo }}{\text { Total de litros de diesel consumidos no ciclo }}
$$

Os custos de manutenção de locomotivas para o caso analisado são: (i) R \$ 0,0012/l para o Grupo 10; (ii) $R \$ 0,0065 / 1$ para o Grupo 9; (iii) $R \$ 0,047 /$ para o Grupo 8; e (iv) $R \$ 0,0717 / 1$ para o Grupo 7. Este cálculo foi realizado com base no histórico dos gastos com manutenção dos últimos três anos das locomotivas agrupadas e, também, no histórico de abastecimento de cada uma. Observa-se que o patamar de custo dos Grupos 9 e 10 é inferior ao dos Grupos 7 e 8, devido à idade da frota e à necessidade distinta de intervenções preventivas e corretivas.

\subsection{Etapa 5: Matriz de Eficiência Energética}

Existem duas formas de se medir o consumo de uma locomotiva: pelo abastecimento da bomba (consumo real) ou pelas informações do registrador de eventos. A primeira forma é simples quando se tem controle de abastecimento e quando a locomotiva é fixa em um único trem tipo, que não altera a rota, não vai para oficinas e não sofre avarias. Com isso, o controle de abastecimento tem de estar atrelado ao histórico de serviços das locomotivas, para que seja possível atribuir a quantidade de litros gasta para cada trabalho realizado sem distorções.

No segundo caso, é necessário acesso aos dados do registrador de eventos da locomotiva. Estes dados são contínuos e remontam todo tipo de operação praticada nas viagens da locomotiva. 0 acelerador das locomotivas varia por pontos de 0 ou Idle a T8, sendo T8 a maior potência disponibilizada e Idle sem tração, porém com um pequeno consumo de diesel. 0 fabricante fornece o valor consumido para ponto de aceleração em fração de tempo. Coletar esta informação e montar a matriz de consumo é uma tarefa difícil e trabalhosa, sendo que, em alguns casos, não se tem histórico de dados. Assim, deve-se optar por estimar a Eficiência Energética $(E E)$ de uma locomotiva em um trem tipo $i$ por (i) ferramentas de simulação de condução de trens, com os parâmetros configurados de forma mais realista possível; ou (ii) estimando estatisticamente através do uso de dados de trens com características similares e que circulam pela mesma rota ou similar.

Para o caso analisado, a matriz de EE foi obtida com dados de viagens, associados com os dados dos registradores de eventos das locomotivas, coletados e armazenados através de um sistema wi-fi instalado nas margens da ferrovia. Outro sistema faz varreduras no parâmetro do acelerador, cruzando com as informações do fabricante, assim, obtendo o consumo das viagens. Dados de 116.198 viagens dos trens escolhidos foram coletados no primeiro semestre de 2016 e tratados com auxílio de planilhas eletrônicas, sendo excluídos erros de medição, não-conformidades e outliers. Como a $E E$ é dada pelos litros consumidos por $T K B$ transportado, essa informação foi obtida para cada trem tipo escolhido. 
O principal desafio desta etapa se deve ao fato de que locomotivas em posições diferentes em uma mesma composição possuem consumos diferentes, pois, geralmente, em operação com a tração distribuída, o maquinista opera com comandos diferentes em cada locomotiva. Logo, foram coletados apenas dados das viagens com informação completa da composição. A Tabela 4 apresenta a eficiência energética dos grupos tratores por trem tipo considerados neste trabalho.

Tabela 4 - Eficiência Energética dos grupos tratores por trem tipo (em Litros x $10^{3}$ /TKB)

\begin{tabular}{ccccc}
\hline \multirow{2}{*}{ Trem Tipo } & \multicolumn{4}{c}{ Grupo trator } \\
\cline { 2 - 5 } & $\mathbf{1 0}$ & $\mathbf{9}$ & $\mathbf{8}$ & $\mathbf{7}$ \\
\hline FER & 2,454 & 3,150 & 5,211 & 5,462 \\
\hline JDN1 & 4,066 & 6,698 & 5,852 & 6,134 \\
\hline JDN2 & 3,832 & 5,355 & 6,333 & 6,638 \\
\hline JDN3 & 3,953 & 3,052 & 5,867 & 6,150 \\
\hline JDN5 & 3,759 & 5,255 & 5,919 & 6,204 \\
\hline JDU & 3,872 & 5,412 & 5,658 & 5,930 \\
\hline NEXP & 2,259 & 2,777 & 5,614 & 5,884 \\
\hline NEV & 2,317 & 2,605 & 5,266 & 5,519 \\
\hline AUX-FA & 2,085 & 2,130 & 4,306 & 4,840 \\
\hline AUX-SM & 3,177 & 3,246 & 4,447 & 5,000 \\
\hline NEZ & 2,571 & 3,398 & 5,860 & 6,588 \\
\hline TOD & 2,419 & 3,185 & 4,365 & 4,907 \\
\hline HPN & 3,619 & 4,766 & 9,002 & 10,120 \\
\hline QVL & 3,303 & 3,550 & 4,865 & 5,469 \\
\hline
\end{tabular}

\subsection{Etapa 6: Informações de planejamento de transportes}

A quantidade de composições necessárias é dimensionada por três fatores: (i) volume de carga contratado pelo cliente, (ii) quantidade de vagões que cada composição levará e, (iii) tempo que cada composição demora na realização de um ciclo inteiro até que seja carregado novamente. Considerando quantidades fixas de vagões por composição, quanto menor for o tempo de ciclo, mais composições serão necessárias para suprir o volume contratado e, quanto mais rápido, menor a quantidade de composições necessárias. Para este trabalho, foram adotadas as informações de TKB e de quantidade de composições $\theta$ contidas na Tabela 1.

\subsection{Etapa 7: Alocação dos Grupos de Tração}

Para a alocação dos grupos de tração, propõe-se um modelo de otimização cujo objetivo é a minimização dos custos, sendo baseado no consumo de combustíveis e no custo de manutenção, uma vez que, de acordo com VALEC (2012), estes são os principais fatores que encarecem a operação das locomotivas. Os parâmetros deste modelo são apresentados na Tabela 5, sendo a variável de decisão $\left(X_{i j}\right)$ referente à quantidade de locomotivas de cada grupo trator que deve ser alocada em cada trem tipo. 
Tabela 5 - Resumo das variáveis e parâmetros do modelo

\begin{tabular}{cll}
\hline Variável & Definição & Unidade \\
\hline$X_{i j}$ & Quantidade de locomotivas do grupo trator $j$ no trem tipo $i$ & $\begin{array}{l}\text { Variável de decisão correspondente } \\
\text { ao número de locomotivas }\end{array}$ \\
\hline Parâmetros & Definição & Unidade \\
\hline$C_{D}$ & Custo do litro de óleo diesel & $\mathrm{R} \$$ \\
\hline$C_{U M j}$ & Custo unitário de manutenção para cada locomotiva do grupo trator $j$ & $\mathrm{R} \$$ litro \\
\hline$E E_{i j}$ & $E E$ do grupo trator $j$ no trem tipo $i$ & litros $/ 1000 \times T K B$. \\
\hline$T K B_{i}$ & $T K B$ total tracionada pelos trens tipo $i$ & Ton. bruta $\times$ km \\
\hline$\theta_{i}$ & Quantidade de composições no trem tipo $i$ & Composições \\
\hline$\theta_{i j}$ & Quantidade de composições no trem tipo $i$ tracionadas pelas locomoti- & Composições \\
\hline$L$ & vas do grupo trator $j$ & Litros \\
\hline$l_{i j}$ & Total de diesel consumido & Quantidade de locomotivas do grupo trator $j$ necessárias para tracionar \\
\hline$L o c_{j}$ & uma composição do trem tipo $i$ & Locomotivas \\
\hline
\end{tabular}

\subsubsection{Modelo}

Analisando a diversidade dos trens em uma ferrovia, suas diferenças de trajeto (distância percorrida) e de volume transportado (TKB), consumo de diesel e tipos de locomotivas utilizadas, é possível entender que cada trem tipo terá uma eficiência energética $(E E)$ diferente para cada do grupo trator. Sendo $j$ um elemento correspondente a um grupo trator formado por uma ou mais locomotivas, então $j \in J$, onde $J$ é o conjunto que representa todos os agrupamentos de locomotivas da frota. Analogamente, $i$ é um elemento correspondente a um trem tipo, sendo que $i \in I$, onde $I$ é o conjunto dos vários trens tipo. Com esses conjuntos definidos, a eficiência energética da ferrovia é calculada pela Equação (2). A $E E$ total da ferrovia pode ser expandida pelo somatório de litros $(L)$ e de $T K B$ de cada trem tipo.

$$
E E=\frac{L}{T K B} \times 10^{3}
$$

onde $\quad E E: \quad$ eficiência energética global da ferrovia;

$L: \quad$ total de litros de diesel consumidos; e

TKB: $\quad$ tonelada bruta $\mathrm{x} \mathrm{km}$ percorrido.

Supondo um trem tipo específico e sua $E E$, a Equação (3) indica o total de litros de combustível consumidos pelo trem tipo.

$$
L=E E \times T K B \times 10^{-3}
$$

Considerando-se os diversos grupos de tração que formam as composições, pode-se aplicar o somatório do consumo de todos os trens tipos $i$, variando, agora, os grupos tratores $j$, conforme a Equação (4), para se calcular o total de litros de combustível consumido na ferrovia.

$$
L=\sum_{i \in \mathbf{I}} \sum_{j \in \boldsymbol{J}} E E_{i j} \times T K B_{i j} \times 10^{-3}
$$

onde $\quad E E_{i j}: \quad$ eficiência energética do grupo trator j no trem tipo i; e

$T K B_{i j}: \quad$ TKB realizado pelo grupo trator $\mathrm{j}$ no trem tipo i.

Uma composição consegue transportar determinada TKB em determinado tempo. Logo, deve haver uma quantidade de composições $\left(\theta_{i}\right)$ circulando simultaneamente na malha para que o plano de transporte $\left(T K B_{i}\right.$ planejado) seja atendido no período mensal.

Considerando que a variável de decisão do modelo é $X_{i j}$ - que representa a quantidade de locomotivas do grupo trator $j$ que devem ser alocadas ao trem tipo $i$, e que $l_{i j}$ é a quantidade de 
locomotivas do grupo trator $j$ necessária para tracionar uma composição do trem tipo $i$ (Quadro de Blocagem dos Grupos Tratores definido anteriormente), então pode-se dividir $X_{i j}$ por $l_{i j} \mathrm{e}$ obter a quantidade de composições do trem tipo $i$ tracionadas por locomotivas do tipo $j\left(\theta_{i j}\right)$. Portanto, o $T K B_{i j}$ é a fração da $T K B_{i}$ tracionada por locomotivas do grupo trator $j$. Ou ainda, a $T K B_{i j}$ é a fração das composições do trem tipo $i$ tracionadas por locomotivas do grupo trator $j$ multiplicado pela $T K B_{i}$ (Equação 5).

$$
T K B_{i j}=\frac{x_{i j} / l_{i j}}{\theta_{i}} \times T K B_{i}
$$

Logo, substituindo a Equação (5) no termo da Equação (4), tem-se a Equação (6).

$$
L=\sum_{i \in \boldsymbol{I}} \sum_{j \in J} E E_{i j} \times \frac{X_{i j}}{\theta_{i} \times l_{i j}} \times T K B_{i} \times 10^{-3}
$$

Como as manutenções preditivas são realizadas com base na quantidade de litros de diesel consumidos, o custo unitário de manutenção $C_{U M j}$ e o custo real do diesel $C_{D}$ são considerados no modelo conferindo valor financeiro.

Assim, como o objetivo do modelo proposto é minimizar custo na ferrovia, utilizando-se os custos indicados, obtém-se a função objetivo do modelo proposto (Equação 7).

$$
\text { Min }_{\text {CustoTotal }}=\sum_{i=1} \sum_{j=1} X_{i j} \times\left(C_{D}+C_{U M j}\right) \times E E_{i j} \times \frac{T K B_{i}}{\theta_{i} \times l_{i j}} \times 10^{-3}
$$

Sujeita às restrições:

$$
\begin{gathered}
\sum_{i \in \mathrm{I}} X_{i j} \leq \operatorname{Loc}_{j} \quad \forall j \in J \\
\sum_{j \in J} \frac{X_{i j}}{l_{i j}}=\theta_{i} \quad \forall i \in I \\
X_{i j}=0 \quad \forall(i, j) \in R \mid R \subset I \times J \\
X_{i j} \in Z^{+} \quad \forall i \in I, j \in J
\end{gathered}
$$

A Função Objetivo (7) está sujeita às restrições de quantidade de locomotivas disponíveis por grupo trator (conjunto de Restrições (8)). Ao somar todas as locomotivas de um determinado grupo trator $j$ alocadas em todos os trens tipo $i$ existentes, deve-se encontrar um número menor que a quantidade total disponível do respectivo grupo. Portanto, existe uma restrição deste tipo para cada grupo $j$ existente.

As Restrições (9) dizem respeito à quantidade de composições necessárias para cada trem tipo. Sabendo-se que o Quadro de Blocagem foi calculado e representado pela Tabela 3, juntamente com o parâmetro $l_{i j}$, ao se dividir a variável de decisão $X_{i j}$ por $l_{i j}$, encontra-se a quantidade de composições que poderão ser tracionadas com as quantidades determinadas em $X_{i j}$. Esta quantidade deve ser igual à quantidade de composições determinadas pelo planejamento de atendimento ao volume do período. Caso seja menor, não atenderá a demanda, e caso seja maior, haverá desperdício de recursos, formando mais composições do que o necessário. Essa restrição garante que se atinja a quantidade de composições determinadas para o trem tipo.

As Restrições (10) referem-se à impossibilidade de determinadas locomotivas passarem por certas rotas. Isso se dá, por exemplo, pela falta de determinado equipamento instalado, gabarito de túneis, peso por eixo acima do permitido para a via permanente e outros motivos quaisquer. 
Para tal, é definido um subconjunto $R$, que representa esta relação binária de $I$ em $J$ que pode restringir combinações de $i$ e $j$ específicas para cada caso de aplicação. A Restrição (11) está relacionada ao domínio das variáveis de decisão.

A modelagem leva em conta apenas os trens que podem ser considerados cíclicos - definidos na Subseção 3.1, como forma de garantir que as locomotivas tracionem sempre um mesmo trem em uma mesma rota. Considerar outros trens implica enfrentar o maior problema apontado pelos autores pesquisados, que é o de scheduling. Nesta modalidade, os horários e rotas dos trens tem que ser considerados pátio a pátio, para que em determinado pátio a alocação de um grupo trator em um trem que vai partir seja atendida em tempo hábil pelo grupo que está em outro trem que ainda vai chegar, considerando tempos de manobra, tempos de espera, recursos do pátio e garantia de lotação. Os modelos que consideraram o problema de scheduling possuem tempos de solução impraticáveis, onde, em vários casos, os inputs se alteram antes que a resposta seja fornecida. Além disso, nas ferrovias brasileiras, trens agendados são menos frequentes, já que os principais produtos transportados são commodities, principalmente, granéis. 0 primeiro passo na modelagem é, portanto, a seleção dos trens tipo.

\subsection{Etapa 8: Alocação dos trens não cíclicos}

Por fim, caso existam trens que não se enquadraram na condição de cíclicos e não podem ser introduzidos no modelo matemático, estes devem ser alocados manualmente às locomotivas que sobrarem.

\section{IMPLEMENTAÇÃO COMPUTACIONAL E RESULTADOS}

A implementação do modelo matemático proposto para alocação dos grupos de tração (seção 3.7) foi realizada no software AIMMS 4.21.5, sendo resolvida como um problema de programação linear inteira (PI) por meio do solver CPLEX 12.6.3. Os dados apresentados nas etapas da Seção 3, como os trens tipo, as rotas (combinações de origem e destino) e demais informações levantadas e exibidas nas Tabelas de 1 a 4, foram utilizados na aplicação do procedimento proposto para análise da alocação de locomotivas a trens de carga geral e/ou de heavy haul, em uma ferrovia brasileira de transporte de carga. A aplicação se baseia em dados de um dos maiores operadores ferroviários do país. Com malha ferroviária de $1.643 \mathrm{~km}$ nos estados da região Sudeste do Brasil, esse operador transporta cargas diversificadas como contêineres, siderúrgicos, cimento, bauxita, agrícolas, coque, carvão e minério de ferro, operando em mais de 100 terminais intermodais e cinco portos.

Atualmente, a ferrovia realiza a alocação de locomotivas de forma manual, sem a ajuda de quaisquer ferramentas, considerando alguns critérios e regras básicas, onde as locomotivas de maior potência são alocadas: (i) aos trens mais pesados (maior volume) e (ii) aos clientes com maior volume. Desta forma, os trens de minério são sempre priorizados em detrimento aos trens de carga geral, recebendo as locomotivas mais modernas e com maior capacidade (Grupos 9 e 10). As demais locomotivas são alocadas conforme histórico de uso da ferrovia.

No caso em análise, o terminal Pederneiras liga a hidrovia Tietê-Paraná à ferrovia que conduz açúcar e produtos agrícolas em grandes volumes até o porto de Santos. Porém, as locomotivas do grupo trator 10 não podem circular no trecho com destino ou origem a este terminal, devido a uma restrição de via permanente que as limita pelo seu peso por eixo. Com isso, as restrições indicadas em (10), sendo $R=\{(4,1),(6,1)\}$, foram adotadas e apresentadas nas Equações (12) e (13). 


$$
\begin{aligned}
& X_{41}=0 \\
& X_{61}=0
\end{aligned}
$$

Depois de inseridas todas as restrições, deve-se determinar a disponibilidade mensal de locomotivas a ser adotada no modelo. Esse é um parâmetro fundamental no planejamento mensal do processo de alocação de locomotivas, porém sofre variações conforme plano de manutenção, novas aquisições, vendas e remanejamentos. Em um determinado mês, por exemplo, algumas locomotivas podem não estar disponíveis, havendo sido alocadas para tração de trens em outras ferrovias, estarem paradas em oficinas, realizando manobras em pátios ou a serviço das equipes de manutenção de via permanente. Adotou-se um total de 342 locomotivas para esse parâmetro, dado que essa foi a média mensal para o último ano.

Tradicionalmente, o planejamento de alocação mensal é realizado manualmente. Nessa situação, denominada de Caso1 (caso base ou business as usual - BAU), tem-se que, para a disponibilidade de 342 locomotivas, o custo mensal de manutenção e combustível é de aproximadamente $\mathrm{R} \$ 43,47$ milhões para executar a quantidade de TKB mensal informada na Tabela 1.

Considerando a mesma quantidade de locomotivas do Caso 1 (342) e demais dados mensais, adotou-se o modelo matemático proposto para realizar a alocação por trem tipo. Para fins de identificação, denomina-se o resultado dessa análise (otimização) como Caso 2. Os resultados obtidos para o Caso 2 indicam um custo mensal de manutenção e combustível de R 42,37 milhões, o que gerou uma economia de $\mathrm{R}$ \$ 1,097 milhões mensais. Portanto, a adoção do modelo matemático e do procedimento proposto permitiu a alocação de locomotivas de forma mais eficiente do que as habituais regras adotadas pela ferrovia.

Ainda, é importante entender os efeitos das variações na disponibilidade de locomotivas em relação às decisões de utilização do modelo para minimizar o custo operacional. Essas análises são importantes, pois ajudam a responder questões como (i) é possível e/ou mais eficiente utilizar menos locomotivas?; (ii) é mais eficiente disponibilizar mais locomotivas?; (iii) no caso de novas aquisições ou substituição de frota, quais modelos devem ser adquiridos e quais devem ser substituídos? Portanto, analisou-se a sensibilidade da resposta (custo) em relação a variações do parâmetro de entrada (quantidade de locomotivas).

Como os Grupos 7 e 8 tendem a ser menos eficientes, foi aplicada a redução nestes grupos para verificar a resposta do modelo com a expectativa de conseguir cumprir o volume com maior eficiência. Em seguida, aplicou-se também a redução em todos os grupos (variando de 5 a 40\%) para efeito de comparação. Diante dos percentuais de redução aplicados, só houve solução pelo modelo com redução de até 5\%. No Caso 3, em que foi analisada a redução de 5\% em todos os tipos de locomotiva, o custo operacional passou a $\mathrm{R} \$ 43,56$ milhões, gerando um aumento de $\mathrm{R}$ \$ 1,2 milhões mensais em comparação ao Caso 2. Por sua vez, a redução de 5\% somente nos Grupos 7 e 8 (Caso 4) gerou um custo operacional de R\$ 42,54 milhões, valor ligeiramente superior ao Caso 2. Desta forma, em resposta a questão (i), entende-se que é possível operar com menos locomotivas, principalmente com Caso 4 que trabalha com oito locomotivas a menos, porém, não é possível superar a economia do Caso 2. Por isso, não é recomendada a redução nos Grupos 9 e 10, apenas nos Grupos 7 e 8 em casos de necessidade e sem expectativas de redução de custo.

0 aumento da disponibilidade das locomotivas também é analisado baseado no incremento arbitrário de $10 \%$ da quantidade de todos os grupos de tratores em quatro casos seguintes (10\%, 20\%, 30\% e 40\%), mantendo-se a demanda de transporte (TKB) apresentada na Tabela 1. 0 objetivo desta vez é responder as questões (ii) e (iii) levantadas previamente. 
Percebe-se que, no caso com $10 \%$ de aumento (Caso 5), o procedimento proposto alocou as 376 locomotivas e, apesar de ser uma quantidade maior, houve economia no custo total, demonstrando um bom aproveitamento destas locomotivas. Nos casos de $20 \%, 30 \%$ e $40 \%$ de aumento (Casos 6, 7 e 8, respectivamente), a disponibilidade de locomotivas adotadas no modelo foi, respectivamente, de 410, 445 e 479. Verifica-se, conforme apresentado na Tabela 6, que os custos operacionais baixaram a cada incremento. Assim, em resposta à questão (ii), é possível uma alocação que garanta menor custo operacional com a disponibilização de mais locomotivas. Porém, para os cenários acima de 10\% (Casos 6, 7 e 8, respectivamente), houve o descarte de algumas locomotivas. Para alguns casos com aumento da quantidade de locomotivas, não foram utilizadas locomotivas do Grupo 7, pois a demanda foi suprida pelos demais grupos. Essa análise responde à questão (iii), em que explorando uma opção de renovação de frota e de novas aquisições, as melhores escolhas são os modelos de Grupos 10 e 9 em detrimento dos Grupos 7 e 8. Não foram destacados mais casos de aumento de disponibilidade devido à manutenção da convergência dos resultados apresentados nestes quatro casos. Assim, os resultados obtidos são apresentados na Tabela 6.

A Figura 3 apresenta as reduções de custos fracionadas em manutenção e combustível para cada caso analisado. Com $10 \%$ de aumento de locomotivas, houve redução mais acentuada no consumo de combustível (litros de diesel), pois se consegue aproveitar as locomotivas extras e alocá-las de forma eficiente. Apesar disso, este aumento de ativos não se reflete de forma tão positiva no custo de manutenção, porém o custo final é menor, compensado pela economia de diesel.

Nas demais variações de percentual de disponibilidade, o custo de manutenção cai de forma mais expressiva devido a menor utilização das locomotivas dos Grupos 7 e 8, que possuem maior custo de manutenção. Porém o consumo de diesel não cai no mesmo patamar, mesmo sendo as locomotivas dos Grupos 9 e 10 mais eficientes que as dos Grupos 7 e 8 . A justificativa é o fato de, por serem dotados de maior potência, os trens mais leves acabam por não ter um aproveitamento completo destas locomotivas devido à sobra de potência. A matriz de EE evidencia este ponto de vista, mostrando que ocorre um aumento do número, ou seja, há uma piora relativa de EE para estas locomotivas em trens de menor peso de arraste.

Nota-se, por meio da Figura 3, que, após o incremento de 10\% na taxa de disponibilidade, os ganhos relativos no custo operacional (custo com combustível e manutenção) são menores, havendo a possibilidade de existir um ponto de corte entre $10 \%$ e $20 \%$. A determinação exata deste ponto depende de uma análise de investimento que pode ser feita a partir do levantamento dos custos do aumento de disponibilidade ou aquisição de mais locomotivas para a frota. Portanto, devem-se analisar as soluções por dois pontos de vista. Quando se tem este excedente de locomotivas em algum local, porém não disponível para a frota, deve-se priorizar a disponibilização das mesmas para que o custo reduza, pois a análise mostra ser vantajoso trabalhar com elas. Contudo, quando não se possui este excedente disponível, deve-se fazer uma análise de investimento, buscando ampliação da capacidade de manutenção e a aquisição ou aluguel/arrendamento desses modelos. 
Tabela 6-Quadro de Resultados

\begin{tabular}{|c|c|c|c|c|c|c|c|c|c|c|c|c|c|c|c|c|}
\hline \multicolumn{2}{|l|}{ Caso } & \multicolumn{4}{|c|}{1} & \multicolumn{3}{|c|}{2} & \multicolumn{3}{|c|}{3} & \multicolumn{5}{|c|}{4} \\
\hline \multirow{3}{*}{\multicolumn{2}{|c|}{ Trem Tipo }} & \multicolumn{4}{|c|}{ Alocação Manual } & \multicolumn{3}{|c|}{ Modelagem } & \multicolumn{3}{|c|}{$\begin{array}{l}\text { Modelagem }-5 \% \text { de } \\
\text { todos os grupos }\end{array}$} & \multicolumn{5}{|c|}{$\begin{array}{c}\text { Modelagem }-5 \% \text { dos } \\
\text { Grupos } 7 \text { e } 8\end{array}$} \\
\hline & & \multicolumn{4}{|c|}{ Grupo trator } & \multicolumn{3}{|c|}{ Grupo trator } & \multicolumn{3}{|c|}{ Grupo trator } & \multicolumn{5}{|c|}{ Grupo trator } \\
\hline & & 10 & 9 & 8 & 7 & 10 & 8 & 7 & 10 & 8 & 7 & 10 & 9 & 8 & 7 & \\
\hline \multicolumn{2}{|c|}{$\begin{array}{c}\text { Total de Locomotivas Inseridas na } \\
\text { Modelagem }\end{array}$} & \multicolumn{4}{|c|}{342} & \multicolumn{3}{|c|}{342} & \multicolumn{3}{|c|}{323} & \multicolumn{5}{|c|}{334} \\
\hline FER & & & & 15 & & & & 15 & & & 15 & & & 0 & 15 & \\
\hline JDN1 & & & & & 12 & & 10 & & & 10 & & & & 10 & & \\
\hline JDN2 & & & & & 24 & & 20 & & & 20 & & & & 20 & & \\
\hline JDN3 & & & & 25 & 18 & & 35 & 6 & 8 & 25 & 6 & & & 35 & 6 & \\
\hline JDN5 & & & & 5 & & & 5 & & & 5 & & & & 5 & & \\
\hline JDU & & & & 8 & & & & 10 & & 4 & 5 & & & 4 & 5 & \\
\hline NEXP & & 62 & 62 & & & 84 & & & $59 \quad 65$ & & & 77 & 47 & & & \\
\hline NEV & & 8 & & & & 1 & & & 3 & & & & 12 & & & \\
\hline AUX-FA & & 31 & 22 & & & 29 & & & 42 & & & 36 & 12 & & & \\
\hline AUX-SM & & & & 12 & & $\epsilon$ & 6 & & 3 & 9 & & & 12 & & & \\
\hline NEZ & & 6 & & & & & 9 & & & 9 & & & 1 & 8 & & \\
\hline TOD & & & & 12 & & 4 & & & 4 & & & 4 & & & & \\
\hline HPN & & & & 10 & & & & 10 & & & 10 & & & & 10 & \\
\hline QVL & & 10 & & & & & 2 & 13 & & & 15 & & & & 15 & \\
\hline $\begin{array}{c}\text { Total de Locomotivas Aloca } \\
\text { grupo } \\
\end{array}$ & las por & 117 & 84 & 87 & 54 & 117 & 87 & 54 & 111 & 82 & 51 & 117 & 84 & 82 & 51 & \\
\hline Total de Locomotivas Alo & adas & & 34 & & & & & & & 323 & & & & 334 & & \\
\hline Custo da Operação & & & 43.4 & 9.178 & & $\mathrm{R} \$ 42$ & 71.653 & & $\mathrm{R} \$ 43$ & 565.7 & & & $\mathrm{R} \$ \mathrm{~S}_{4}$ & 2.547. & & \\
\hline Caso & & 5 & & & & & & & & 7 & & & & 8 & & \\
\hline Trem Tino & $\begin{array}{r}\text { Mod } \\
\text { to }\end{array}$ & $\begin{array}{l}\text { lagem } \\
\text { los os }\end{array}$ & $\begin{array}{l}+10 \% \\
\text { rupos }\end{array}$ & & Moc & $\begin{array}{r}\text { delagem }+ \\
\text { os gr }\end{array}$ & $\%$ de $t$ & odos & $\begin{array}{r}\text { Mode } \\
\text { tod }\end{array}$ & $\begin{array}{l}\text { agem } \\
\text { os os g }\end{array}$ & $\begin{array}{l}30 \% d \\
\text { upos }\end{array}$ & & $\begin{array}{r}\text { Mode } \\
\text { toc }\end{array}$ & $\begin{array}{l}\text { lagem } \\
\text { los os } \xi\end{array}$ & $\begin{array}{l}40 \% d \\
\text { upos }\end{array}$ & \\
\hline Trem про & & rupot & ator & & & Grupo & ator & & & upo tr & tor & & & rupo t & tor & \\
\hline & 10 & 9 & 8 & 7 & 10 & 9 & 8 & 7 & 10 & 9 & 8 & 7 & 10 & 9 & 8 & 7 \\
\hline $\begin{array}{c}\text { Total de Locomotivas } \\
\text { Inseridas na Modelagem }\end{array}$ & & 376 & & & & 4 & & & & 445 & & & & 479 & & \\
\hline FER & & & 2 & 13 & & & 14 & 1 & & & 15 & & & 10 & & \\
\hline JDN1 & & & 10 & & & & 10 & & & & 10 & & & & 10 & \\
\hline JDN2 & & & 20 & & 3 & & 15 & & 12 & & & & 12 & & & \\
\hline JDN3 & & & 10 & 36 & & 2 & 35 & 3 & & 12 & 25 & & & 32 & & \\
\hline JDN5 & & & 5 & & & & 5 & & & & 5 & & & & 5 & \\
\hline JDU & & & & 10 & & & & 10 & & & 8 & & & & 8 & \\
\hline NEXP & 124 & & & & 124 & & & & 124 & & & & 124 & & & \\
\hline NEV & & 12 & & & & 12 & & & 8 & & & & 8 & & & \\
\hline AUX-FA & 2 & 80 & & & 9 & 66 & & & 4 & 76 & & & 13 & 58 & & \\
\hline AUX-SM & & & 12 & & & 12 & & & & 12 & & & & 12 & & \\
\hline NEZ & & & 9 & & & 9 & & & & 9 & & & 2 & 6 & & \\
\hline TOD & 3 & & 3 & & 4 & & & & 4 & & & & 4 & & & \\
\hline HPN & & & 10 & & & & 10 & & & & 10 & & 1 & & 8 & \\
\hline QVL & & & 15 & & & & 15 & & & & 15 & & & & 15 & \\
\hline $\begin{array}{l}\text { Total de Locomotivas } \\
\text { Alocadas por grupo }\end{array}$ & 129 & 92 & 96 & 59 & 140 & 101 & 104 & 14 & 152 & 109 & 88 & 0 & 164 & 118 & 46 & 0 \\
\hline $\begin{array}{c}\text { Total de Locomotivas } \\
\text { Alocadas }\end{array}$ & & $37 €$ & & & & 3 & & & & 349 & & & & 328 & & \\
\hline Custo da Operação & & 40.46 & 5.804 & & & $\mathrm{R} \$ 39.6$ & 4.827 & & & 39.01 & 053 & & & 38.43 & 022 & \\
\hline
\end{tabular}




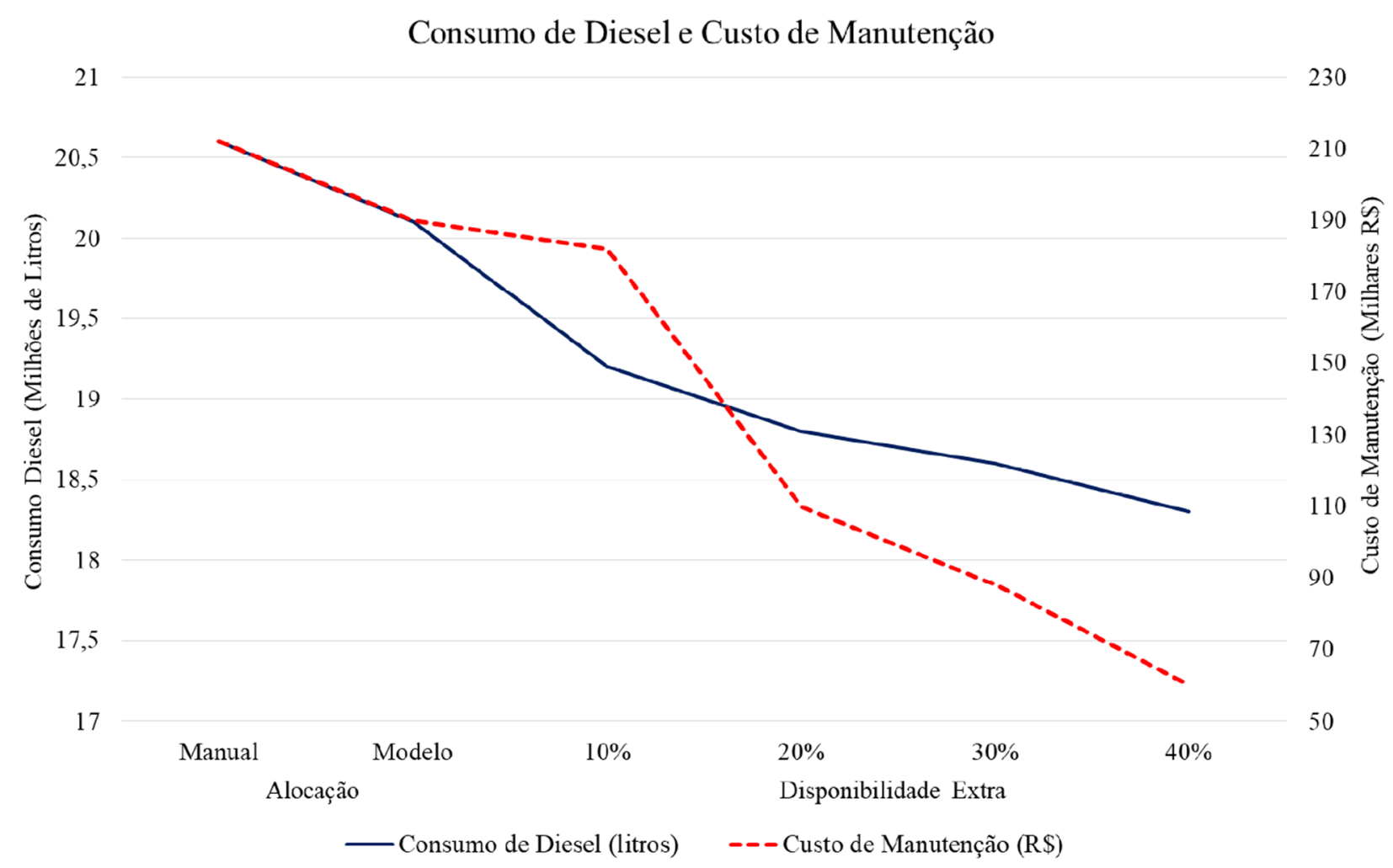

Figura 3. Análise de sensibilidade do consumo de diesel e custo de manutenção

\section{CONSIDERAÇÕES FINAIS}

Este trabalho buscou apresentar uma forma de avaliar e propor, de acordo com os trabalhos de maior relevância publicados a respeito do assunto, um procedimento para a alocação de frota de locomotivas aos trens de carga, feito a partir da coleta e análise de dados estatísticos, para a obtenção de uma resposta que considere a redução de dois dos principais responsáveis pelo custo de operação de locomotivas: combustível e manutenção. 0 diferencial do procedimento proposto é que ele não busca resolver o problema totalmente através de um modelo de otimização, tornando assim o problema mais simples, trazendo análises e particularidades dos casos para as etapas de preparação até a aplicação do modelo. Desta forma, foram obtidos tempos de solução compatíveis para o cenário de uma ferrovia real e flexibilidade para alterações dos principais parâmetros. 0 desenvolvimento do procedimento partiu da metodologia de custeio das operações ferroviárias das locomotivas e das teorias de dimensionamento de tração para os trens. $\mathrm{O}$ ponto de partida da modelagem matemática foi a formulação de Eficiência Energética, utilizada por ferrovias do mundo todo, agregando ao contexto os demais parâmetros que influenciam no custo de operação. Esta é mais uma grande vantagem deste trabalho, pois o procedimento foi proposto pautado em uma linguagem genérica e internacional, sendo adaptável a qualquer ferrovia de carga que possua os dados indicados.

A análise dos resultados revelou que, comparando a alocação manual e a alocação obtida com a aplicação do procedimento proposto, foi possível um ganho de $\mathrm{R} \$ 1,097$ milhões mensais. A análise de sensibilidade ao aumento da disponibilidade mostrou que os ganhos em custos operacionais não superam os investimentos necessários para os aumentos de frota apresentados, não demonstrando viabilidade financeira. Porém, indica os benefícios de uma eventual necessidade de renovação de frota. 
Em termos de limitações, o procedimento trata de forma diferente os trens não cíclicos. Contudo, é importante destacar que, para o caso abordado, os trens cíclicos foram responsáveis por mais de $90 \%$ da $T K B$ da ferrovia em questão, de modo que os trens não cíclicos não trazem tanto impacto ao custo total. Deste modo, sugere-se como oportunidade para trabalhos futuros a inclusão de trens não cíclicos para a eliminação da etapa final, tornando o procedimento mais completo.

\section{REFERÊNCIAS}

Ahuja, R.K.; J. Liu; J.B. Orlin; D. Sharma e L.A Shughart (2005) Solving Real Life Locomotive Scheduling Problems. Transportation Science, v. 39, p. 503-517. DOI: https://doi.org/10.1287/trsc.1050.0115

Cassemiro, S.L.S. (2017) Procedimento de Planejamento de Alocação de Locomotivas. Dissertação Mestrado em Engenharia de Transportes. Instituto Militar de Engenharia, Rio de Janeiro.

Florian, M.; G. Bushell; J. Ferland; G. Guerin e L. Nastansky (1976) The Engine Scheduling Problem in a Railway Network. INFOR: Information System and Operational Research, v.14, p. 121-138. DOI: https://doi.org/10.1080/03155986.1976.11731632

Gualda, N. D. F. e L.M.S.F. Murgel (1999) Modelo para Formação de Composições Ferroviárias. Transportes, v.7, p. 30-63. DOI: https://doi.org/10.14295/transportes.v7i1.219

Hu, H.; K. Li e X. Xu (2013). A Multi-objective Train Scheduling Optimization Model Considering Locomotive Assignment and Segment Emission Constraints for Energy Saving. Journal of Modern Transportation, v.21, p.9-16. DOI: https://doi.org/10.1007/s40534-013-0003-1

Louzada, P.C.; R.A. Rosa; M.B. Foletto; L.B. Pimenta (2018) Estratégia de Utilização da Folga de Tração de Trens para Planejamento da Distribuição de Vagões Vazios. Transportes, v.26, p. 155 - 166. DOI: https://doi.org/10.14295/transportes.v26i2.1537

Magalhães, P.C.B. (2001) Módulo VII - Fundamentos de Logística Empresarial: Ênfase na Atividade Transporte. Pós-Graduação em Engenharia Ferroviária. PUC-MG, Belo Horizonte.

MRS Logística S.A. (2014) Malha Ferroviária e Frota. Disponível em https://www.mrs.com.br/empresa/ferrovia-frota/. Acesso em: 01 de Agosto de 2018.

Paoletti, B. e S. Cappelletti (2007) The Optimization Model for Locomotive Rostering in the Fleet Planning Project. The Proceedings of the Multiconference on Computational Engineering in Systems Applications. Institute of Electrical and Electronics Engineers-IEEE, Beijing, China. p. 362-365. DOI: https://doi.org/10.1109/cesa.2006.4281679

Piu, F. e M.G. Speranza (2013) The Locomotive Assignment Problem: A Survey on Optimization Models. International Transaction in Operational Research, v.21, p. 327-352.

DOI: https://doi.org/10.1111/itor.12062

Piu, F.; V. Kumar; M. Bierlaire e M.G. Speranza (2015) Introducing a Preliminary Consists Selection in the Locomotive Assignment Problem. Transportation Research, v.82, p. 217-237. DOI: https://doi.org/10.1016/j.tre.2015.07.003

Su, R.; L. Zhou e J. Tang (2015) Locomotive Schedule Optimization for Da-qin Heavy Haul Railway. Hindawi Publishing Corporation Mathematical Problems in Engineering, v.2015, Article ID 607376. DOI: https://doi.org/10.1155/2015/607376

VALEC (2012) Elaboração de Estudo de Viabilidade Técnica, Econômica e Ambiental (EVTEA) para Implantação de Trechos Ferroviários da EF-151: Segmento 2 - Ligação Goiânia/Anápolis/Brasília, Recife. 